\title{
Prognostic factors in Chinese patients with metastatic castration-resistant prostate cancer treated with docetaxel-based chemotherapy
}

\author{
Yuan-Yuan Qu ${ }^{1,2, *}$, Bo Dai ${ }^{1,2, *}$, Yun-Yi Kong ${ }^{2,3}$, Ding-Wei Ye ${ }^{1,2}$, Xu-Dong Yao ${ }^{1,2}$, Shi-Lin Zhang ${ }^{1,2}$, \\ Hai-Liang Zhang ${ }^{1,2}$, Chun-Guang $\mathrm{Ma}^{1,2}$ and Wei-Yi Yang ${ }^{1,2}$
}

This study aims to evaluate the potential value of patient characteristics in predicting overall survival (OS) in patients with metastatic castration-resistant prostate cancer (mCRPC) treated with docetaxel-based thermotherapy. A total of 115 patients with mCRPC undergoing a docetaxel q3w regimen were enrolled in this study. A survival analysis was performed using the Kaplan-Meier method. Cox proportional hazards models were used to evaluate the prognostic value of all covariates for OS. OS was also analysed after stratifying patients according to the results of multivariate analysis. The median OS for the entire cohort was $\mathbf{1 7 . 0}$ months. The multivariate analysis showed that the prostate-specific antigen doubling time (PSADT), baseline haemoglobin ( $\mathrm{Hb}$ ) concentration, alkaline phosphatase (ALP) concentration, cycles of chemotherapy and time to castration resistance were independent prognostic factors of OS. According to the presence of PSADT $<\mathbf{4 6 . 3}$ days and baseline ALP $\geqslant 110 \mathrm{IU} \mathrm{I}^{-1}$, all patients were divided into three risk groups: low-risk group (no risk factors), intermediate-risk group (one risk factor) and high-risk group (two risk factors). Median OSs for patients in low-, intermediate- and high-risk groups were 28.0 months (95\% Cl: 23.8-32.2), 21.0 months (95\% Cl: 18.9-23.1) and 11.0 months (95\% Cl: 7.6-14.4), respectively $(P<0.001)$. In conclusion, PSADT, baseline $\mathrm{Hb}$ concentration, ALP concentration, cycles of chemotherapy and time to castration resistance were independent prognostic factors of OS in Chinese patients with mCRPC treated with docetaxel. PSADT combined with the baseline ALP concentration could be a useful risk stratification parameter for evaluating survival outcomes.

Asian Journal of Andrology (2013) 15, 110-115; doi:10.1038/aja.2012.110; published online 12 November 2012

Keywords: castration-resistant; docetaxel; metastatic; overall survival; prognostic factor; prostate cancer

\section{INTRODUCTION}

Prostate cancer is the most frequently occurring cancer in men from Western countries and represents the second leading cause of cancerrelated death, next to lung cancer. ${ }^{1}$ In China, the incidence of prostate cancer has increased dramatically over the past two decades, most likely due to economic development and lifestyle changes. ${ }^{2}$ Moreover, most newly diagnosed Chinese prostate cancer patients already have metastatic disease because prostate cancer screening using prostatespecific antigen (PSA) and digital rectal examination is not a routine practice in China. $^{2}$

Androgen deprivation therapy is the initial treatment for metastatic prostate cancer and leads to improvement in bone pain, regression of soft tissue metastases, and a reduction in serum levels of PSA. ${ }^{3}$ Unfortunately, in virtually all patients, the disease inevitably advances to the androgen-independent stage within a median of 18-24 months after castration. ${ }^{4}$ Two randomized phase III studies, SWOG 99-16 ${ }^{4}$ and TAX $327,{ }^{5}$ demonstrated that docetaxel-based chemotherapy confers a significant survival benefit of $20 \%-24 \%$ in patients with metastatic castration-resistant prostate cancer (mCRPC) compared with a mitoxantrone regimen. ${ }^{6}$ Nevertheless, many patients failed to respond to docetaxel chemotherapy and experienced considerable toxicity. Currently, the identification of readily available prognostic factors would be an essential step in optimising the management of patients being treated with docetaxel.

Several prognostic factors in patients with mCRPC have been reported, including Eastern Cooperative Oncology Group performance status (ECOG PS), baseline serum PSA level, haemoglobin $(\mathrm{Hb})$, alkaline phosphatase (ALP), lactate dehydrogenase (LDH), prostate-specific antigen doubling time (PSADT), time to castration resistance and circulating tumor cells. ${ }^{7-14}$ However, the magnitude of the benefit provided by each factor has varied among studies, and the value of these factors in predicting overall survival (OS) for Chinese patients is still unclear. In the present study, we retrospectively analysed the data from 115 mCRPC patients treated with docetaxel to explore the prognostic factors of survival outcomes in Chinese patients. 


\section{MATERIALS AND METHODS}

\section{Patients and variables}

The present study is based on data from 115 consecutive Chinese patients with mCRPC who received docetaxel-based chemotherapy at a standard dose and schedule 5 at our institution from November 2005 to September 2011. These patients were diagnosed between October 1997 and July 2010. All eligible patients had histologically confirmed adenocarcinoma of the prostate and confirmed cases of mCRPC, which was determined by three sequential rises in serum PSA level with castrate levels of serum testosterone $\left(<50 \mathrm{ng} \mathrm{dl}^{-1}\right)$. All patients presented with metastatic disease(s) upon imaging (computed tomography, magnetic resonance imaging or whole body radionuclide bone scan). The time to castration resistance was calculated from the time of diagnosis until the confirmation of CRPC. The present study was approved by the Institutional Review Board of our institution, and written informed consent was obtained from each patient before enrolment.

We retrospectively collected the variables, including patient characteristics at diagnosis (age, serum PSA level, Gleason score and metastatic site(s)) and characteristics at the start of chemotherapy (age, serum PSA level, PSADT, complete blood count, biochemistry profile, ECOG PS, metastatic site(s), radical prostatectomy, cycles of chemotherapy and time to castration resistance). All laboratory analyses were performed in the same laboratory.

\section{Treatment}

All patients were treated on a 21-day cycle, with $75 \mathrm{mg} \mathrm{m}^{-2}$ of docetaxel (Taxotere, Aventis, Essex, UK) administered intravenously on the first day and $5 \mathrm{mg}$ of prednisone administered twice daily throughout the cycle. On days 1-3, dexamethasone was administered at a dosage of $10 \mathrm{mg}$ twice daily to prevent potential acute hypersensitivity reactions or residual body fluid. The average number of chemotherapy cycles was 6 (range: 1-16).

\section{Definition and calculation of PSADT}

By definition, PSADT was defined as the time required for the PSA level to double. PSADT was calculated from the slope of the linear regression calculated from the natural logarithm values of all serial PSA (on the ordinate) versus time of measurement (on the abscissa), according to the following formula: PSADT $=(\ln 2 \times T) /\left[\ln \left(\mathrm{PSA}_{2}\right)-\right.$ $\left.\ln \left(\mathrm{PSA}_{1}\right)\right]$, where $\ln$ is the natural $\log , T$ is the time (in days) between PSA measurements, $\mathrm{PSA}_{2}$ is the PSA defining CRPC and PSA $\mathrm{A}_{1}$ is the next most recent PSA level. ${ }^{15}$

\section{The end point of the present study}

The end point of the present study was the effect of patient characteristics on OS, calculated as the interval between the first day of docetaxel administration and the date of death or the last follow-up visit for censored (living) patients.

\section{Statistical analysis}

The number of chemotherapy cycles was classified according to the mean value, baseline gamma-glutamyl transpeptidase (GGT) and $\mathrm{LDH}$ concentrations were classified according to the upper limit of normal range and baseline $\mathrm{Hb}$ concentration was classified according to the cut-off point of $110 \mathrm{~g} \mathrm{l}^{-1}$. All other characteristics were divided into dichotomous variables according to the median value. A survival analysis was performed using the Kaplan-Meier method, and the differences in OS rates were assessed by the log-rank test. The relationship between a given explanatory covariate and the survival data was evaluated using a univariate Cox regression analysis. Only variables which were statistically significant in the univariate analysis $(P<0.05)$ were included in the multivariate model. A multivariate Cox proportional hazards regression analysis was used to analyse the results while controlling for important prognostic covariates that could potentially confound the analyses. OS was also analysed after stratifying patients by PSADT and baseline ALP concentration according to the results of the multivariate Cox regression analysis. For all statistical tests, a twosided $P$ value of less than 0.05 was considered statistically significant. All statistical analyses were performed using SPSS software version 16.0 (SPSS Inc., Chicago, IL, USA).

\section{RESULTS}

\section{Patient characteristics}

Follow-up continued until 30 April 2012. During the follow-up period (median: 45.0 months; range: $2.0-74.0$ months), 87 of 115 patients (75.6\%) died, and all deaths were attributed to prostate cancer or prostate cancer-related complications. The median OS for the entire cohort was 17.0 months (95\% CI: 13.8-20.2; range: $2.0-46.0$ months). The Gleason scores were not available for 46 patients who came from rural areas and were diagnosed at local hospitals so long ago that the pathological specimens could not be obtained. The demographics and disease characteristics for all patients were summarized in Table 1.

\section{Univariate and multivariate Cox regression analysis for OS}

Kaplan-Meier and log-rank tests were used to assess OS and compare differences in survival time distributions among patient characteristics with respect to OS (Table 2).

The results of univariate and multivariate Cox regression analysis for OS in 115 patients were summarized in Table 3. The univariate analysis of baseline clinical characteristics identified a total of nine statistically significant predictors of OS: baseline serum PSA level, PSADT, baseline $\mathrm{Hb}$ concentration, ALP concentration, GGT concentration, LDH concentration, ECOG PS, cycles of chemotherapy and time to castration resistance. Subsequent multivariate analysis reserved PSADT $(P=0.005 ; \mathrm{HR}=0.407)$, baseline $\mathrm{Hb}$ concentration $(P=0.028 ; \quad H R=0.495)$, baseline ALP concentration $(P=0.019$; $\mathrm{HR}=1.934)$, cycles of chemotherapy $(P<0.001 ; \mathrm{HR}=0.379)$ and time to castration resistance $(P=0.030$; $\mathrm{HR}=0.548)$ as independent prognostic factors of OS. The median OS for patients with PSADT $<46.3$ days and $\geqslant 46.3$ days were 14.0 months (95\% CI: 10.5-17.5) and 23.0 months (95\% CI: 19.9-26.1), respectively $(P=0.001)$. OS curves were reported in Figure 1. Median OS for patients with ALP $\geqslant 110 \mathrm{IU} \mathrm{l}^{-1}$ and $<110 \mathrm{IU} \mathrm{l}^{-1}$ were 15.0 months (95\% CI: $\left.12.1-17.9\right)$ and 26.0 months (95\% CI: $21.0-30.9)$, respectively $(P<0.001)$. OS curves were reported in Figure 2.

\section{Risk stratification according to PSADT and baseline ALP concentration}

According to the presence of PSADT $<46.3$ days and baseline ALP $\geqslant 110 \mathrm{IU}^{-1}$, which were two of most statistically significant risk factors in the multivariate analysis, we divided the entire cohort into the following three risk groups: low-risk group (no risk factors), intermediate-risk group (one risk factor, either PSADT $<46.3$ days or ALP $\geqslant 110 \mathrm{IU} \mathrm{l}^{-1}$ ) and high-risk group (two risk factors, both PSADT $<46.3$ days and ALP $\geqslant 110 \mathrm{IU}^{-1}$ ). The median OSs for patients in low-, intermediate- and high-risk groups were 28.0 months (95\% CI: 23.8-32.2), 21.0 months (95\% CI: 18.9-23.1) and 11.0 months (95\% CI: 7.6-14.4), respectively. The hazard ratios of intermediate- and high-risk to low-risk were 1.927 (95\% CI: 1.093-3.398) and 10.143 
Table 1 Patient characteristics at diagnosis and at the initiation of chemotherapy in 115 mCRPC patients treated with docetaxal

\begin{tabular}{|c|c|}
\hline Characteristics & Median \\
\hline \multicolumn{2}{|l|}{ Characteristics at diagnosis } \\
\hline Age (year) & $65(49-81)$ \\
\hline PSA $\left(n g \mathrm{ml}^{-1}\right)$ & $100(2.9-3034.4)$ \\
\hline \multicolumn{2}{|l|}{ Gleason score (No. of patients, \%) } \\
\hline$\leqslant 6$ & $5(4.3)$ \\
\hline 7 & $21(18.3)$ \\
\hline 8 & $15(13.0)$ \\
\hline 9 & $22(19.1)$ \\
\hline 10 & $6(5.2)$ \\
\hline Not available & $46(40.0)$ \\
\hline \multicolumn{2}{|l|}{ Metastatic site(s) (No. of patients, \%) } \\
\hline $\mathrm{M}_{0}$ & 24 (20.9) \\
\hline$M_{1 a}$ & $21(18.3)$ \\
\hline$M_{1 b}$ & $68(59.1)$ \\
\hline$M_{1 c}$ & $2(1.7)$ \\
\hline \multicolumn{2}{|l|}{ Characteristics at the start of chemotherapy } \\
\hline Age (year) & $68(51-82)$ \\
\hline PSA (ng ml $\left.{ }^{-1}\right)$ & $90.5(0.1-4066.4)$ \\
\hline PSADT (day) & $46.3(5.6-661.1)$ \\
\hline WBC $\left(\times\left. 10^{9}\right|^{-1}\right)$ & $6.4(3.2-14.7)$ \\
\hline $\operatorname{PTL}\left(\times\left. 10^{9}\right|^{-1}\right)$ & $165(72-518)$ \\
\hline $\mathrm{Hb}\left(\mathrm{g} \mathrm{I}^{-1}\right)$ & $121(68-150)$ \\
\hline Albumin $\left(\left.g\right|^{-1}\right)$ & $43.1(31.2-50.7)$ \\
\hline $\operatorname{ALP}\left(I \cup I^{-1}\right)$ & $110(33-1083)$ \\
\hline GGT $\left(I \cup I^{-1}\right)$ & $33(11-217)$ \\
\hline $\mathrm{LDH}\left(\mathrm{IU} \mathrm{I}^{-1}\right)$ & $234(124-1693)$ \\
\hline \multicolumn{2}{|l|}{ ECOG PS (No. of patients, \%) } \\
\hline 0 & $40(34.8)$ \\
\hline 1 & $65(56.5)$ \\
\hline 2 & $10(8.7)$ \\
\hline \multicolumn{2}{|l|}{ Metastatic site(s) (No. of patients, \%) } \\
\hline Lymph node & 39 (33.9) \\
\hline Bone & $115(100.0)$ \\
\hline Viscera & $16(13.9)$ \\
\hline Lung & $9(7.8)$ \\
\hline Bladder & $4(3.5)$ \\
\hline Liver & $2(1.7)$ \\
\hline Brain & $1(0.9)$ \\
\hline \multicolumn{2}{|l|}{ RP (No. of patients, \%) } \\
\hline Yes & $12(10.4)$ \\
\hline No & $103(89.6)$ \\
\hline Cycles of chemotherapy & $5(1-16)$ \\
\hline Time to castration resistance (month) & $23(2-114)$ \\
\hline
\end{tabular}

Abbreviations: ALP, alkaline phosphatase; ECOG PS, Eastern Cooperative Oncology Group performance status; GGT, gamma-glutamyl transpeptidase; $\mathrm{Hb}$,

haemoglobin; LDH, lactate dehydrogenase; mCRPC, metastatic castration-resistant prostate cancer; PLT, platelet; PSA, prostate-specific antigen; PSADT, prostatespecific antigen doubling time; RP, radical prostatectomy; WBC, white blood cell.

(95\% CI: 3.982-25.836), respectively. The OS curves according to risk stratification were distinctly tiered and statistically significant $(P<0.001)$, as shown in Figure 3.

\section{DISCUSSION}

This retrospective observation study demonstrates the significant value of several prechemotherapeutic variables, including PSADT, baseline $\mathrm{Hb}$ concentration, baseline ALP concentration, cycles of chemotherapy and time to castration resistance, as independent prognostic factors of OS in patients with mCRPC treated with docetaxel-based chemotherapy.
The PSADT, which assumes that tumour growth is logarithmic, is a simple tool used to determine the growth rate of the serum PSA level. There are several reports available regarding the prognostic impact of PSADT for prostate cancer patients. ${ }^{10,16,17}$ D'Amico et al. ${ }^{16}$ suggested that the PSADT should be confirmed as a useful surrogate marker for survival and as a tool to identify patients at risk of progression after radical prostatectomy or radiotherapy. Teeter et al. ${ }^{17}$ reported that in an older, racially diverse cohort, a short PSADT (less than 9 months) on recurrence after radical prostatectomy was associated with worse all-cause mortality. Recently, an analysis of a TAX327 cohort indicated that baseline PSADT was an independent predictor of survival, demonstrating that patients with a short PASDT (less than 55 days) had a lower chance of survival compared with patients who had a more indolent PSADT. ${ }^{10}$ As mentioned above, our study also demonstrated that a rapid increase in the serum PSA level, namely, a short PSADT, is independently associated with a negative survival outcome. The median OSs for patients with PSADT $<46.3$ days and those with PSADT $\geqslant 46.3$ days were 14.0 months and 23.0 months, respectively $(P=0.001)$. Consequently, the PSADT was a valuable tool capable of selecting high-risk patients before the onset of chemotherapy. It could be used to assess prognosis after relapse and to guide therapeutic strategy in patients being treated with docetaxel.

According to the inclusion criteria, all 115 patients developed bone metastases. Bone metastases from prostate cancer are characterized by both excessive bone formation and resorption due to the increased number and activity of osteoblasts and osteoclasts. ${ }^{18}$ Total serum ALP, a routinely and successively measured marker that is inexpensive and readily available, could partly reflect osteoblastic activity. Although total serum ALP is a relatively nonspecific biomarker and can be elevated by either bone or liver metastasis, patients with bone metastasis and an elevated baseline ALP are likely to have bone as the dominant source of ALP. ${ }^{8}$ Additionally, patients with mCRPC rarely have liver metastasis that could cause ALP elevations (only 2 of 115 patients had liver metastasis in this study). A study of 643 patients indicated that the serum levels of total ALP and bone-specific alkaline phosphatase were correlated highly and that total ALP primarily reflected bone-specific alkaline phosphatase in men with mCRPC and bone metastasis. ${ }^{18}$ In large multivariate analyses of patients with metastatic androgen-independent prostate cancer, elevated levels of serum total ALP were independently associated with shorter OS. ${ }^{19,20}$ Our current results also indicted that patients with baseline serum total ALP $\geqslant 110 \mathrm{IU}^{-1}$ had shorter OS than patients with baseline serum total ALP $<110 \mathrm{IU} \mathrm{l}^{-1}$ (15.0 months vs. 26.0 months, $P<0.001)$. Baseline ALP $\geqslant 110 \mathrm{IU}^{-1}$ predicted a nearly two-fold increased risk of death $(\mathrm{HR}=1.934)$.

To our knowledge, no study to date has been performed to clarify whether PSADT, combined with baseline ALP concentration, contributes to independent prognostic information for OS in MCRPC patients. Our study demonstrated that patients with a short PSADT and a high baseline ALP concentration were at the highest risk of decreased OS, patients with a long PSADT and a low baseline ALP concentration were at the lowest risk of death, and patients with one of the two risk factors fell into the intermediate-risk category. This suggests that mCRPC patients can be risk-stratified according to PSADT and baseline ALP concentration. Therefore, when estimating the prognosis for mCRPC patients being treated with docetaxel, both PSADT and ALP concentration should be calculated in order to provide more accurate prognostic information. Furthermore, we suggest that the combination of a short PSADT and a high baseline ALP concentration 
Table 2 Summary of the association between patient characteristics and overall survival (OS) for all patients

\begin{tabular}{|c|c|c|c|c|}
\hline Characteristics & No. of patients & No. of deaths & Median of OS (month) & Log-rank test $\mathrm{P}$ \\
\hline All patients & 115 & 87 & 17.0 & - \\
\hline \multicolumn{5}{|l|}{ Characteristics at diagnosis } \\
\hline Age (year) & & & & 0.726 \\
\hline$\geqslant 65$ & 64 & 52 & 15.0 & \\
\hline PSA (ng ml ${ }^{-1}$ ) & & & & 0.790 \\
\hline$<100$ & 40 & 29 & 20.0 & \\
\hline Yes & 91 & 70 & 14.0 & \\
\hline No & 24 & 17 & 18.0 & \\
\hline \multicolumn{5}{|l|}{ Characteristics at the start of chemotherapy } \\
\hline Age (year) & & & & 0.548 \\
\hline$<68$ & 55 & 37 & 15.0 & \\
\hline$\geqslant 68$ & 60 & 50 & 18.0 & \\
\hline$<46.3$ & 57 & 48 & 14.0 & \\
\hline$\geqslant 46.3$ & 58 & 39 & 23.0 & \\
\hline WBC $\left(\times\left. 10^{9}\right|^{-1}\right)$ & & & & 0.552 \\
\hline$<6.4$ & 56 & 46 & 19.0 & \\
\hline$\geqslant 6.4$ & 59 & 41 & 17.0 & \\
\hline $\operatorname{PTL}\left(\times\left. 10^{9}\right|^{-1}\right)$ & & & & 0.824 \\
\hline$<165$ & 57 & 48 & 16.0 & \\
\hline$\geqslant 165$ & 58 & 39 & 20.0 & \\
\hline $\mathrm{Hb}\left(\mathrm{g} \mathrm{I}^{-1}\right)$ & & & & 0.002 \\
\hline$<110$ & 28 & 24 & 10.0 & \\
\hline$\geqslant 110$ & 87 & 63 & 19.0 & \\
\hline Albumin $\left(\left.g\right|^{-1}\right)$ & & & & 0.252 \\
\hline$<43.1$ & 57 & 47 & 15.0 & \\
\hline$<250$ & 71 & 44 & 21.0 & \\
\hline$\geqslant 250$ & 46 & 43 & 14.0 & \\
\hline ECOG PS & & & & 0.002 \\
\hline 0 & 40 & 21 & 24.0 & \\
\hline $1-2$ & 75 & 66 & 15.0 & \\
\hline Metastatic site(s) & & & & 0.680 \\
\hline Bone only & 99 & 73 & 17.0 & \\
\hline Bone and viscera & 16 & 14 & 13.0 & \\
\hline $\mathrm{RP}$ & & & & 0.060 \\
\hline Yes & 12 & 7 & 25.0 & \\
\hline No & 103 & 80 & 16.0 & \\
\hline Cycles of chemotherapy & & & & $<0.001$ \\
\hline$<6$ & 59 & 55 & 12.0 & \\
\hline$\geqslant 6$ & 56 & 32 & 24.0 & \\
\hline Time to castration resistance (month) & & & & 0.006 \\
\hline$<23$ & 57 & 48 & 14.0 & \\
\hline$\geqslant 23$ & 58 & 39 & 21.0 & \\
\hline
\end{tabular}

Abbreviations: ALP, alkaline phosphatase; ECOG PS, Eastern Cooperative Oncology Group performance status; GGT, gamma-glutamyl transpeptidase; Hb, haemoglobin; LDH, lactate dehydrogenase; PLT, platelet; PSA, prostate-specific antigen; PSADT, prostate-specific antigen doubling time; RP, radical prostatectomy; WBC, white blood cell. 
Table 3 Summary of the association between patient characteristics and overall survival: Cox univariate and multivariate regression analysis

\begin{tabular}{|c|c|c|c|c|c|c|}
\hline \multirow{2}{*}{ Characteristics } & \multicolumn{3}{|c|}{ Univariate analysis } & \multicolumn{3}{|c|}{ Multivariate analysis } \\
\hline & $P$ & $H R$ & $95 \% \mathrm{Cl}$ & $P$ & $H R$ & $95 \% \mathrm{Cl}$ \\
\hline \multicolumn{7}{|l|}{ Characteristics at diagnosis } \\
\hline PSA & 0.791 & 1.069 & $0.652-1.754$ & & & \\
\hline Metastases & 0.063 & 1.571 & $0.817-3.168$ & & & \\
\hline \multicolumn{7}{|c|}{ Characteristics at the start of chemotherapy } \\
\hline PSADT & 0.002 & 0.501 & $0.326-0.770$ & 0.005 & 0.407 & $0.237-0.701$ \\
\hline WBC & 0.499 & 1.183 & $0.727-1.927$ & & & \\
\hline PLT & 0.942 & 0.982 & $0.589-1.613$ & & & \\
\hline $\mathrm{Hb}$ & 0.005 & 0.469 & $0.278-0.792$ & 0.028 & 0.495 & $0.265-0.925$ \\
\hline Albumin & 0.256 & 0.715 & $0.400-1.276$ & & & \\
\hline ALP & $<0.001$ & 2.157 & $1.400-3.321$ & 0.019 & 1.934 & $1.112-3.363$ \\
\hline Cycles of chemotherapy & $<0.001$ & 0.335 & $0.214-0.535$ & $<0.001$ & 0.379 & $0.225-0.639$ \\
\hline Time to castration resistance & 0.007 & 0.557 & $0.365-0.855$ & 0.030 & 0.548 & $0.318-0.943$ \\
\hline
\end{tabular}

Abbreviations: ALP, alkaline phosphatase; Cl, confidence interval; ECOG PS, Eastern Cooperative Oncology Group performance status; GGT, gamma-glutamyl transpeptidase; Hb, haemoglobin; HR, hazard ratio; LDH, lactate dehydrogenase; PLT, platelet; PSA, prostate-specific antigen; PSADT, prostate-specific antigen doubling time; RP, radical prostatectomy; WBC, white blood cell.

could be used as a guide for the early initiation of chemotherapy for patients with mCRPC.

Previous studies have demonstrated that the baseline PSA level, which is generally considered to be related to the tumour burden, could be a prognostic factor of OS for mCRPC patients. ${ }^{9}$ In our study, an elevated baseline PSA level was shown to be associated with shorter OS in univariate analysis. However, the multivariate analysis failed to confirm baseline PSA level as an independent prognostic factor of OS $(P=0.407)$. This might be a result of the relatively small sample size and the possible selection bias, as patients with ECOG PS greater than score 2 were excluded in this study.

In the present study, we identified prognostic factors using only prechemotherapeutic variables, which are easily measured by standard assays in most institutions. This facilitates the oncologist's ability to predict the survival outcomes for mCRPC patients before the initiation of chemotherapy. However, potential flaws in the current study

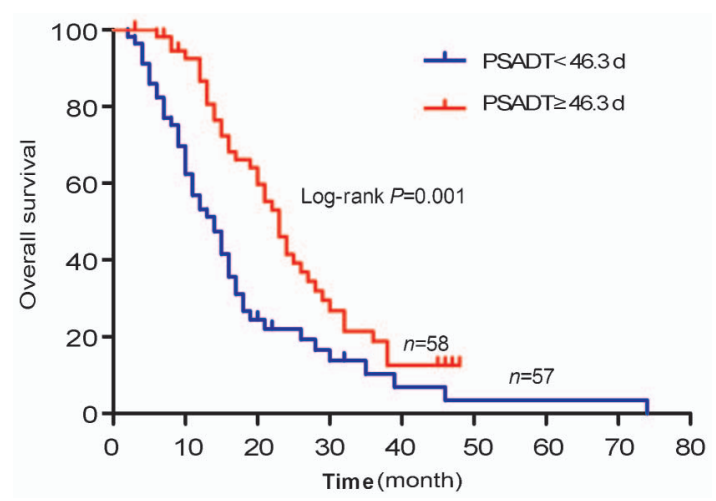

Figure 1 Overall survival curves for $115 \mathrm{mCRPC}$ patients treated with docetaxel, divided into PSADT $<46.3$ days and PSADT $\geqslant 46.3$ days groups. mCRPC, metastatic castration-resistant prostate cancer; PSADT, prostate-specific antigen doubling time. include its retrospective nature and the relatively small sample size. Future prospective studies should be performed to validate our results in a larger population of patients.

\section{CONCLUSIONS}

In conclusion, this retrospective analysis demonstrated that PSADT, baseline $\mathrm{Hb}$ concentration, baseline ALP concentration, cycles of chemotherapy and time to castration resistance could provide independent prognostic information on OS in Chinese patients with mCRPC treated with docetaxel. These variables can be used to optimize the management of mCRPC. In addition, PSADT combined with baseline ALP concentration could be a useful risk stratification parameter for evaluating survival outcomes.

\section{AUTHOR CONTRIBUTIONS}

YYQ and BD designed the study, collected, analysed and interpreted the clinical data, wrote and revised the manuscript. YYK reviewed

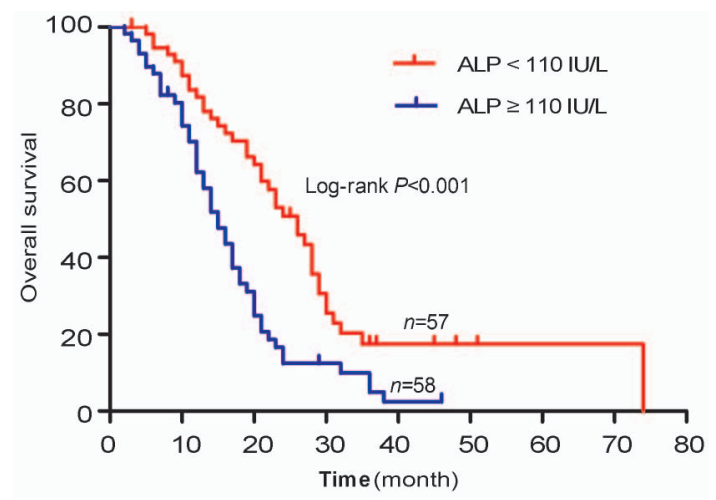

Figure 2 Overall survival curves for $115 \mathrm{mCRPC}$ patients treated with docetaxel, divided into ALP $<110 \mathrm{IU} \mathrm{I}^{-1}$ and ALP $\geqslant 110 \mathrm{IU} \mathrm{I}^{-1}$ groups. ALP, alkaline phosphatase; $\mathrm{mCRPC}$, metastatic castration-resistant prostate cancer. 


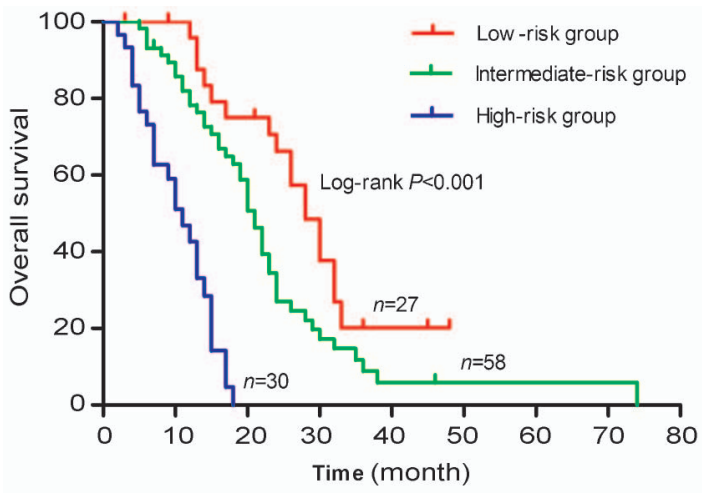

Figure 3 Overall survival curves for $115 \mathrm{mCRPC}$ patients treated with docetaxel according to the risk groups. According to the presence of PSADT $<46.3$ days and/or ALP $\geqslant 110 \mid \mathrm{IU} \mathrm{I}^{-1}$, each patient was divided into low-, intermediate- and high-risk groups. ALP, alkaline phosphatase; mCRPC, metastatic castrationresistant prostate cancer; PSADT, prostate-specific antigen doubling time.

pathological slides and revised the manuscript. DWY supervised the project and revised the manuscript. XDY, SLZ, HLZ, CGM and WYY collected partial patients' clinical data and followed up with patients. All the authors vouched for the respective data and analysis, approved the final version and agreed to publish the manuscript.

\section{COMPETING FINANCIAL INTERESTS}

The authors declare that they have no financial or commercial interests related to this study.

\section{ACKNOWLEDGMENTS}

This study was supported in part by grants from National Natural Science Foundation of China (Nos. 30801149 and 30973009).

1 Ferlay J, Autier P, Boniol M, Heanue M, Colombet M et al. Estimates of the cancer incidence and mortality in Europe in 2006. Ann Oncol 2007; 18: 581-92.

2 Dai B, Ye DW, Kong YY, Shen YJ, Wang BH. Individualized prostate biopsy strategy for Chinese patients with different prostate-specific antigen levels. Asian J Andro/ 2008 10: 325-31.
3 Crawford ED, Eisenberger MA, McLeod DG, Spaulding JT, Benson R et al. A controlled trial of leuprolide with and without flutamide in prostatic carcinoma. $N$ Engl J Med 1989; 321: 419-24.

4 Petrylak DP, Tangen CM, Hussain MH, Lara PN, Jones JA et al. Docetaxel and estramustine compared with mitoxantrone and prednisone for advanced refractory prostate cancer. N Engl J Med 2004; 351: 1513-20.

5 Tannock IF, de Wit R, Berry WR, Horti J, Pluzanska A et al. Docetaxel and prednisone or mitoxantrone plus prednisone for advanced prostate cancer. N Engl J Med 2004; 351: 1502-12.

6 Petrylak DP. The treatment of hormone-refractory prostate cancer: docetaxel and beyond. Rev Urol 2006; 8(Suppl 2): 48-55.

7 He J, Zeng ZC, Yang P, Chen B, Jiang W et al. Clinical features and prognostic factors for patients with bone metastases from prostate cancer. Asian J Androl 2012; 14: 505-8.

8 Sonpavde G, Pond GR, Berry WR, de Wit R, Armstrong AJ . Serum alkaline phosphatase changes predict survival independent of PSA changes in men with castration-resistant prostate cancer and bone metastasis receiving chemotherapy. Urol Oncol 2010; e-pub ahead of print 29 Sep 2010; doi:10.1016/j.urolonc. 2010.07.002

9 Saad F, Segal S, Eastham J. Prostate-specific antigen kinetics and outcomes in patients with bone metastases from castration-resistant prostate cancer treated with or without zoledronic acid. Eur Urol 2012; e-pub ahead of print 12 May 2012 doi:10.1016/j.eururo.2012.05.007.

10 Armstrong AJ, Garrett-Mayer ES, Yang YC, de Wit R, Tannock IF. A contemporary prognostic nomogram for men with hormone-refractory metastatic prostate cancer: a TAX327 study analysis. Clin Cancer Res 2007; 13: 6396-403.

11 Bournakis E, Efstathiou E, Varkaris A, Wen S, Chrisofos M et al. Time to castration resistance is an independent predictor of castration-resistant prostate cancer survival. Anticancer Res 2011; 31: 1475-82.

12 de Bono JS, Scher HI, Montgomery RB, Parker C, Miller MC et al. Circulating tumor cells predict survival benefit from treatment in metastatic castration-resistant prostate cancer. Clin Cancer Res 2008; 14: 6302-9.

13 Darshan MS, Loftus MS, Thadani-Mulero M, Levy BP, Escuin D et al. Taxane-induced blockade to nuclear accumulation of the androgen receptor predicts clinical responses in metastatic prostate cancer. Cancer Res 2011; 71: 6019-29.

14 Armstrong AJ, Eisenberger MA, Halabi S, Oudard S, Nanus DM et al. Biomarkers in the management and treatment of men with metastatic castration-resistant prostate cancer. Eur Urol 2012; 61: 549-59.

15 Pound CR, Partin AW, Eisenberger MA, Chan DW, Pearson JD. Natural history of progression after PSA elevation following radical prostatectomy. JAMA 1999; 281 $1591-7$.

16 D'Amico AV, Cote K, Loffredo M, Renshaw AA, Schultz D. Determinants of prostate cancer-specific survival after radiation therapy for patients with clinically localized prostate cancer. J Clin Onocol 2002; 20: 4567-73.

17 Teeter AE, Presti JC Jr, Aronson WJ, Terris MK, Kane CJ et al. Does PSADT after radical prostatectomy correlate with overall survival?-a report from the SEARCH database group. Urology 2011; 77: 149-53.

18 Cook RJ, Coleman R, Brown J, Lipton A, Major P et al. Markers of bone metabolism and survival in men with hormone-refractory metastatic prostate cancer. Clin Cancer Res 2006; 12: 3361-7.

19 Smaletz O, Scher HI, Small EJ, Verbel DA, McMillan A et al. Nomogram for overall survival of patients with progressive metastatic prostate cancer after castration. J Clin Oncol 2002; 20: 3972-82.

20 Halabi S, Small EJ, Kantoff PW, Kattan MW, Kaplan EB et al. Prognostic model for predicting survival in men with hormone-refractory metastatic prostate cancer. J Clin Oncol 2003; 21: 1232-7. 